\title{
Proposição do modelo PLM-PV3G para a gestão do ciclo de vida de produtos
}

\author{
Proposal of the PLM-PV3G model for product lifecycle \\ management
}

ISSN 0104-530X (Print) ISSN 1806-9649 (Online)

\author{
Paulo Sergio Gonçalves de Oliveira ${ }^{1}$ \\ André Luis Helleno \\ Dirceu da Silva ${ }^{3}$ \\ Meire dos Santos Lopes ${ }^{4}$ \\ Fernando Celso de Campos ${ }^{2}$ \\ Djair Picchiai ${ }^{5,6}$
}

\begin{abstract}
Resumo: O objetivo do artigo é propor um modelo de gestão de ciclo de vida de produtos no setor metal-mecânico. Essa proposição visa facilitar a adoção de boas práticas no setor, por meio da redução da complexidade e melhoria da assertividade das ações. Para atingir esse objetivo, foi realizado um levantamento com especialistas do setor, com o qual se obteve um total de 397 questionários. O modelo tem como foco principal o projeto de produtos verdes para apoiar a gestão da colaboração, da manufatura e do conhecimento durante a gestão do ciclo de vida de produtos, facilitando, dessa forma, a adoção de tal metodologia. O modelo foi desenvolvido utilizando-se a análise PLS (Partial Least Squares).
\end{abstract}

Palavras-chave: Gestão do ciclo de vida de produtos; Partial Least Squares; Gestão de mudanças; Gestão do conhecimento.

\begin{abstract}
The objective of the article is to propose a model for product lifecycle management in the metalworking sector. This proposal intends to facilitate the adoption of good practices in the sector, by reducing complexity and improving action assertiveness. To achieve this goal, a survey was carried out with specialists from the sector, obtaining a total of 397 questionnaires. The focus of the model is to design green products to give support to the collaboration, manufacturing and knowledge management during product lifecycle management and making it easier to adopt this methodology. The model was developed using PLS (Partial Least Squares) analysis.
\end{abstract}

Keywords: Product lifecycle management; Partial least squares; Change management; Knowledge management.

\section{Introdução}

Nos últimos anos, tem crescido o investimento na área de gerenciamento do ciclo de produtos (PLM) em todos os tipos de empresa, devido, principalmente, ao aumento da complexidade dos produtos e também ao aumento da exigência dos clientes, o que fez com que as empresas começassem a adotar a integração de fornecedores no processo de desenvolvimento de produtos (Tang \& Qian, 2008).

A gestão do ciclo de vida de produtos (Product Lifecycle Management) é uma abordagem para a gestão integrada dos processos de negócio e das informações

relacionadas a esses itens. Tal abordagem requer a utilização de sistemas de informação (Sistemas PLM) para apoiar a colaboração na empresa, ao longo de todo o ciclo de vida (Zancul, 2009).

\section{Gestão do Ciclo de Vida de Produtos (PLM)}

A Gestão do Ciclo de Vida de Produtos (PLM) é definida por Farouk et al. (2008) como uma sistemática integração de todas as informações relacionadas

\footnotetext{
${ }^{1}$ Universidade Anhembi Morumbi, Rua Casa do Ator, 275, Vila Olímpia, CEP 04546-001, São Paulo, SP, Brasil, e-mail: psoliveira@anhembi.br ${ }^{2}$ Universidade Metodista de Piracicaba, Rodovia Luis Ometto, Km 24, CEP 13451-900, Piracicaba, SP, Brasil, e-mail: alhelleno@gmail.com; fccampos@unimep.br

${ }^{3}$ Universidade Nove de Julho, Rua Deputado Salvado Julianelli, CEP 01156-080, São Paulo, SP, Brasil, e-mail: meires1@uninove.br ${ }^{4}$ Universidade Estadual de Campinas, Av. Bertrand Russell, 801, CEP 13083-865, Campinas, SP, Brasil, e-mail: dirceu@g.unicamp.br

${ }^{5}$ Faculdade do Campo Limpo - FACCAMP, Rua Guatemala, 167, CEP 13231-230, Campo Limpo, SP, Brasil, e-mail: djair.picchiai@fgvsp.br ${ }^{6}$ Fundação Getulio Vargas - FGV, Avenida Nove de Julho, 2029, CEP 01313-902, São Paulo, SP, Brasil, e-mail: djair.picchiai@fgvsp.br Recebido em Dez. 01, 2017 - Aceito em Abr. 27, 2018
}

Suporte financeiro: Nenhum. 
ao produto e aos processos durante todo o ciclo de vida, desde a ideia do projeto inicial até o descarte, o que torna a PLM uma estratégia de negócios para suportar uma criação coletiva, gerenciamento, disseminação e o uso das informações do produto na empresa estendida.

O objetivo da PLM é a configuração otimizada dos processos, especialmente do processo de desenvolvimento de produtos, assim como a gestão de todas as informações do produto ao longo do ciclo de vida deste. As informações atualizadas podem ser acessadas diretamente por todas as pessoas autorizadas, a qualquer momento, possibilitando, por exemplo, reduzir o tempo necessário para desenvolver novos produtos com menores custos (Lambert, 2010).

A PLM permite a colaboração criativa, o gerenciamento, a disseminação e o uso da definição do produto e informação dos processos operacionais na empresa estendida, do conceito de marketing até a retirada do produto do mercado (Lee et al., 2008). Em função disso, a PLM foi proposta, nos anos recentes, como uma abordagem de negócios que tem como propósito integrar as pessoas, processos, sistemas de negócio e o gerenciamento da informação no ciclo completo de desenvolvimento do produto entre as empresas.

Dessa forma, pode-se concluir que o (PLM) é uma estratégia de negócio que propõe a gestão consistente de todos os estágios do ciclo de vida do produto, desde o início de sua comercialização até o descarte, envolvendo, dessa forma, grande quantidade de stakeholders (clientes, fornecedores e órgãos reguladores), os quais requerem diversos níveis de detalhes e representações da informação (Thimm et al., 2006).

A Gestão do Ciclo de Vida de Produtos (PLM) será discutida em oito subcapítulos, a seguir.

\subsection{Gestão do desenvolvimento de novos produtos}

Segundo Nisson \& Fagerström (2006), para se desenvolver um produto bem balanceado é preciso considerar a opinião dos stakehoders envolvidos em todo o ciclo de vida do produto.

Outro aspecto fundamental é a especificação dos produtos, que precisa espelhar com a maior fidelidade possível as aspirações dos clientes. Para isso, pode-se utilizar a metodologia QFD (Desdobramento da Função Qualidade), a qual pode ser efetuada em fases, por meio da criação de módulos, com o objetivo de facilitar o gerenciamento.

Para validar as fases do desenvolvimento do produto, pode-se utilizar uma revisão e aprovação formal no final de cada etapa, a qual é chamada de revisão em Gates, de forma a verificar se todos os requisitos foram cumpridos. Tal abordagem pode trazer grandes benefícios para a organização (Rozenfeld \& Forcellini, 2006).
Nantes \& Lucent (2009) afirmam que, para atender às reais necessidades dos clientes, o processo de desenvolvimento de produto (PDP) precisa ser suportado por inovações tecnológicas que diferenciam os produtos no mercado, melhorando a participação da empresa no mercado, o que garante sua sobrevivência.

Quanto mais intensa a incorporação de tecnologia nos produtos, maior é a chance de sucesso destes, se comparados com aqueles com pequena diferenciação e que não incorporam inovações (Gecevska et al., 2010).

Porém, Nantes \& Lucent (2009) alertam que o desenvolvimento de produtos no Brasil caracteriza-se, principalmente, pela melhora de produtos, por meio de pequenas adaptações para adequá-los ao mercado local. Outra característica presente na produção no Brasil é a baixa utilização de universidades e centros de pesquisa, porém, existe um aumento na utilização de parcerias entre os fornecedores de matérias-primas e clientes, caracterizando a inovação como pertencente à empresa e incremental.

Lee et al. (2008) argumentam que este tipo de abordagem, que visa ao aprimoramento de produtos, faz com que surjam diversos desafios, tais como a inovação contínua, a colaboração global e o gerenciamento de riscos complexos. Para o sucesso desse tipo de abordagem, é necessário que os recursos intelectuais, na forma de dados de processos e produtos, estejam acessíveis a qualquer um na cadeia de valor, por meio de uma gestão do conhecimento eficiente, de modo a favorecer a inovação (Trotta, 2010).

\subsection{Envolvimento dos fornecedores e desenvolvimento da cadeia de suprimentos}

As empresas têm adotado a integração dos fornecedores no processo de desenvolvimento de produtos, assim como têm oferecido treinamentos para esses fornecedores como forma de desenvolver a cadeia de suprimentos para obter melhores resultados.

$\mathrm{Na}$ verdade, esse fenômeno tem encontrado fundamentação na pesquisa contemporânea nos campos de engenharia simultânea e gestão da cadeia de suprimentos ( $\mathrm{SCM}$ ), os quais apontam que significantes benefícios podem ser atingidos por meio da integração e envolvimento dos fornecedores nos estágios iniciais de desenvolvimento de produto, também conhecido pela sigla ESI (Tang \& Qian, 2008).

A definição típica de cadeia de suprimentos refere-se a todas as atividades associadas com a transformação e o fluxo de bens e serviços, de fontes materiais em produtos ou serviços para o usuário final, sendo que é a cadeia responsável por gerenciar ambas as atividades internas e externas da organização (Kainuma \& Tawara, 2006).

Tradicionalmente, o conceito de cadeia de suprimentos se refere ao fluxo de transformação dos produtos de baixo para cima, desde a matéria-prima até a entrega do produto para o consumidor final, 
tendo como foco principal o fluxo dos materiais. A cadeia de suprimentos se refere ao gerenciamento das relações entre as empresas, por meio dos processos de negócios, com o objetivo de se criar um fluxo de valor (Santos \& Forcellini, 2009).

As atividades no processo de SCM são influenciadas por diversas variáveis, tais como o tipo de produto, a fase comercial do ciclo de vida do produto, as mudanças demandadas pelos clientes, o lançamento de novas tecnologias, a pressão por regulação, os competidores no mercado, entre outros. Estas variáveis impõem mudanças nos objetivos estratégicos dos processos de negócio, o que faz com que os clientes e fornecedores internos e externos interajam por meio das atividades do processo de negócio. O compartilhamento de informações e conhecimento entre os envolvidos auxilia na criação do sistema de valor, com o objetivo de preencher os diferentes requisitos durante o ciclo de desenvolvimento do produto (Santos \& Forcellini, 2009).

Para gerenciar as relações com os fornecedores, há uma metodologia chamada ESI (Early Supplier Involvement), que verifica a forma como a cooperação externa é realizada entre o desenvolvedor do produto (design) e o desenvolvedor das peças ou módulos. Segundo esta metodologia, as relações se enquadram basicamente em três modalidades (Tang et al., 2004):

- ESI Clássico: refere-se ao relacionamento tradicional cliente-fornecedor, no qual ambas as partes tendem a melhorar suas próprias posições em detrimento da cooperação;

- ESI Modelo I: significa que o fornecedor é integrado à cadeia de processos e, desta forma, contribui com seu conhecimento (know-how) durante todo o ciclo de vida do produto;

- ESI Modelo II: sugere que o cliente (design) é responsável apenas por apresentar as necessidades, enquanto o fornecedor assume as responsabilidades de projetar o produto e as ferramentas, porém, por se tratar de um modelo mais avançado, não pode ser adequado à realidade de muitas empresas, por pressupor um alto grau de confiança e também de maturidade.

Para atingir melhores desempenhos no desenvolvimento conjunto de novos produtos, Sharma (2005) propõe um modelo chamado CPI (Collaborative Product Innovation), que tem como objetivo unificar três práticas: colaboração, desenvolvimento e inovação, as quais, normalmente, são desenvolvidas separadamente.

Nesse sentido, grandes esforços têm sido feitos para solucionar problemas de comunicação entre as áreas da organização e também entre os fornecedores durante o desenvolvimento de novos produtos (NPDI). Em geral, tanto o NPDI quanto o gerenciamento do ciclo de vida de produtos possuem os mesmos desafios no processo de tomada de decisão. A melhoria do processo de decisão ocorre por meio da integração de pessoas e processos em torno do ciclo de vida do produto (Trotta, 2010; Sharma, 2005).

\subsection{Gestão de redes de colaboração e gestão da empresa expandida}

Para superar o desafio de desenvolver produtos globais e também para reduzir o tempo de projeto e os custos de produção, as empresas estão criando centros de projeto espalhados pelo mundo, de forma a aproveitar a expertise regional e também os baixos custos de mão de obra. Tais iniciativas são chamadas de projetos virtuais, nos quais raramente os membros possuem contato físico com os outros participantes do projeto (Brito et al., 2009). Trata-se de equipes virtuais que utilizam tecnologia avançada para o desenvolvimento global de produtos.

Uma das vantagens de se utilizar equipes virtuais é o fato de que um projeto pode estar em andamento durante quase 24 horas por dias, já que contará com colaboradores que estarão trabalhando em diferentes fusos horários. Porém, a desvantagem dessas equipes reside no fato de que esses colaboradores possuem pouco ou nenhum contato físico, o que gera dificuldade em estabelecer relações de confiança, fato que reduz sensivelmente a colaboração.

Apesar de contarem com tecnologias avançadas, as equipes virtuais dependem muito de fatores humanos, de uma forte coordenação e de objetivos de projeto bem definidos para que atinjam o seu máximo potencial.

Muitas empresas têm desenvolvido redes de colaboração entre fornecedores e parceiros, com o objetivo de redução do ciclo de desenvolvimento do produto e das incertezas durante o processo de tomada de decisão, de forma a atender com maior velocidade e precisão as demandas existentes no mercado (Sharma, 2005).

No entanto, para obter melhoria na colaboração, é essencial se ater aos processos de engenharia colaborativa, tendo como foco os seguintes requisitos: promover dados compartilhados, fornecer transparência de localidade, facilitar acesso aos dados de produto, prover notificações aos usuários e monitorar o progresso (Rouibah \& Oud-Ali, 2007).

\subsection{Engenharia simultânea e a utilização de sistemas PDM}

O ambiente global competitivo está colocando pressão no mercado, fato que forçou as organizações a adotarem um grande número de práticas que modificam a natureza da competição, pois o desenvolvimento de novos produtos (PDP) e a engenharia simultânea vêm ganhando, cada vez mais, significância (Possamai et al., 2007). 
Em adição à engenharia simultânea, também existe a melhoria e de certa forma, em paralelo, a ênfase na sincronização da cadeia de suprimentos e a participação nas decisões do desenvolvimento de produtos. Os fatores que motivam esta ênfase são o elevado reconhecimento de muitos fatores inter-relacionados, a terceirização de ambas, manufatura e atividades de projeto (Santos \& Forcellini, 2009).

A engenharia simultânea possui dois recursos essenciais, que são os processos competitivos e a capacidade que as equipes de projetos multifuncionais possuem para puxá-los. Num processo de engenharia simultânea, todos os elementos do desenvolvimento do produto são nomeados, desde o início, como um grupo de atividades e objetivos focados nos anseios dos clientes (Possamai et al., 2007).

Para colaborar com os parceiros e fornecedores, frequentemente existe a necessidade de criação de interfaces entre os sistemas PDM da empresa e dos fornecedores. Os sistemas PDM assumem um papel importante na utilização da engenharia simultânea, pois permitem o compartilhamento de documentos de diversos formatos e também a manutenção do alinhamento estratégico das áreas de desenvolvimento de produtos e manufatura. Dessa forma, a utilização de software PDM é a chave para se atingir a definição do produto digital e também para fornecer conhecimento às empresas parcerias, de modo a permitir a engenharia simultânea (Su \& Liu, 2008).

Como as empresas parceiras normalmente utilizam diferentes tipos de sistemas PDM, é recomendável que usem mecanismos de compartilhamento de arquivos e dados oriundos desses sistemas, sendo uma boa opção a utilização de uma representação em Lightweight programming model.

\subsection{Gestão de mudanças durante o ciclo de vida do produto}

A gestão do ciclo de vida do produto também envolve monitorá-lo após o seu lançamento, e não somente durante o projeto inicial, de forma a se fazerem as mudanças necessárias, permitindo a descontinuidade planejada e incorporação de lições aprendidas durante o ciclo de vida do produto, dentro do processo de desenvolvimento (Brigatini \& Miguel, 2009).

Porém, Rouibah \& Caskey (2003) afirmam que o projeto de um produto em uma empresa, ou num consórcio, é um processo interativo e requer mudança. A habilidade das empresas em gerenciar melhor as mudanças de engenharia (ECs), durante o desenvolvimento do produto, pode reduzir os custos, encurtar o tempo de desenvolvimento e produzir produtos de alta qualidade. No entanto, as mudanças ocorrem durante todo o ciclo de vida do produto e podem ser originadas de diversas fontes, como a utilização de rede de colaboração entre diversas empresas parceiras.
Nesse ambiente colaborativo, é necessário envolver todas as partes, para avaliar o impacto e o controle da mudança e decidir conjuntamente como conduzi-la. $\mathrm{O}$ controle da mudança requer diversas atividades de coordenação, enquanto a avaliação do impacto da mudança requer o gerenciamento do casamento entre os componentes que estão sendo modificados e a interface existente entre os demais componentes ou desenvolvimento das atividades. Ou seja, deve-se gerenciar o relacionamento entre os objetos (peças, componentes, parâmetros etc.) com os objetos que irão sofrer mudanças, observando-se o modo como irão impactar o produto como um todo.

Durante o desenvolvimento de produtos, os engenheiros precisam tomar determinadas decisões sem a criação de documentos ou sem seguir processos. Essas decisões são chamadas variáveis determinadas de engenharia, sendo tais variáveis elementares definidas como parâmetros. Durante um projeto colaborativo, diferentes pessoas decidem sobre os valores dos parâmetros, capturando o relacionamento destes e, consequentemente, especificando o relacionamento entre os tomadores de decisão (Rouibah \& Caskey, 2003).

A seleção de parâmetros a serem gerenciados pode ser decida numa reunião inicial entre os parceiros colaboradores da cadeia de suprimentos. Os parâmetros podem ser distinguidos como funcionais, por exemplo, um motor, uma geometria, ou relacionados ao material, tal como um número de metal. Os parâmetros definidos e declarados para se poder efetuar o gerenciamento de cadeia de fornecimento são categorizados como um sistema de parâmetros e interface de parâmetros (Yang et al., 2004).

Para o gerenciamento dos parâmetros, pode-se utilizar um sistema de avaliação do grau de maturidade destes, que variam numa escala de HG1 até HG5, a qual representa os graus de maturidade entre os departamentos. HG1 se refere a um parâmetro que possui um valor estimado, enquanto HG5 se refere a um parâmetro com valor exato com tolerâncias finais (Rouibah \& Caskey, 2003).

Esse fluxo pode ser automatizado, utilizando-se o conceito de arquitetura orientada a serviços (SOA) e também pelo mapeamento dos processos. Tendo-se como base o modelo BPM, a implementação física pode ser feita utilizando-se o software BPEL (Siller et al., 2008).

Para que as mudanças possam ser propagadas, faz-se necessária a integração de sistemas, tais como o ERP e o PDM. Atualmente, utiliza-se o conceito de criação de tabela para se efetuar a intermediação de dados, devido ao fato de existirem poucas interfaces disponíveis no mercado. Para fazer a troca de dados, também se pode utilizar o conceito de serviços ou SOA (Hou \& Su et al., 2008).

A preocupação em relação ao gerenciamento das mudanças se deve ao fato de os avanços na moderna tecnologia terem resultado em muitos sistemas, produtos e processos complexos, que tornam o 
gerenciamento de projetos muito difícil, aumentando, consideravelmente, a complexidade nas mudanças do projeto, na análise e no gerenciamento, e também no que se refere a todo o ciclo de vida do produto (Venkatasubramanian, 2005).

\subsection{Gestão do conhecimento distribuído durante o ciclo de vida do produto}

Quando estão desenvolvendo produtos globais, muitas empresas procuram por cooperação mútua entre suas unidades ao redor do mundo. Tal cooperação demanda a existência de equipes de projetos nas quais os membros são culturalmente diversos e dispersados geograficamente. Essas circunstâncias introduzem um grande grau de complexidade para a gestão dos projetos, especificamente no que concerne à comunicação e à coordenação, por meio de equipe de projetos multiculturais e dispersas (Livieiro \& Kaminski, 2009).

As interações entre os membros da equipe e a troca de experiências nas resoluções de problemas geram conhecimento, fazendo com que as organizações criem conhecimento dinamicamente. Nonaka et al. (2000) propuseram um modelo que consiste de três elementos, composto por Seci (Socialização, Externalização, Combinação e Internalização), BA e Ativos do Conhecimento.

O gerenciamento eficiente dos dados de engenharia do produto é critico para uma melhoria corporativa efetiva. As empresas estão buscando técnicas e ferramentas que permitirão controlar o projeto, a engenharia e a mensuração, por meio de tecnologias de colaboração (Rouibah \& Oud-Ali, 2007).

Em face da demanda por gestão do conhecimento em projetos e gestão de mudança de produtos, em setembro de 2005 a OMG (Object Management Group) publicou uma RFP (Requisição de proposta) para um padrão internacional de conhecimento baseado em engenharia, chamado de KBE, para a gestão do ciclo de vida de produtos (PLM) (Fan \& Bermell-Garcia, 2008).

$\mathrm{O}$ padrão requer que os sistemas $\mathrm{KBE}$ possuam uma série de funcionalidades, como (Fan \& Bermell-Garcia, 2008):

- Consultar e retornar a geometria e os serviços de geração de topologia, incluindo entrada de dados, saída, mensagens disponíveis e atributos internos do objeto.

- Explicitamente definir os fluxos de entrada e saída de dados, instanciando serviços dentro das aplicações.

A aplicação de gestão do conhecimento é efetuada por meio de processos de configuração, nos quais módulos ou componentes do produto são selecionados e montados, de acordo com a requisição do cliente.
A configuração do produto utiliza, normalmente, grandes volumes de conhecimento, devido às complexas relações existentes entre os componentes, tais como regras de configuração e restrições de montagem (Gecevska et al., 2010; Jinsong et al., 2008).

\subsection{Gestão da manufatura durante o ciclo de vida do produto}

A importância da flexibilidade da manufatura está em proporcionar às empresas uma resposta mais dinâmica às mudanças de mercado e em atender às necessidades dos clientes, sendo que a inabilidade em medi-la dificulta a avaliação dos benefícios produzidos (Gerwin, 2005).

Com o objetivo de se atingir flexibilidade da manufatura, Cui \& Qi (2006) propõem a integração via PLM, criando assim uma plataforma uniforme e integrada, que suporta as operações da empresa, permitindo o gerenciamento dos dados do produto, com velocidade e eficiência, controlando as alterações e também compartilhando as informações entre todos os envolvidos.

A importância do PLM reside no fato de integrar os dados dos sistemas CAx (CAD, CAM, CAIP etc.), ERP, CRM e sistemas SCM (Trotta, 2010). Essa integração possibilita a tomada de decisões mais rápida e também que os gestores avaliem melhor os impactos de mudanças.

Por esse motivo, o PLM é considerado um paradigma que possibilita trazer inovação para a área de manufatura, permitindo às organizações desenvolverem conteúdos de engenharia e integrar todos os processos de negócio, por meio do ciclo completo de vida do produto na empresa estendida (Kim et al., 2008).

Por outro lado, o departamento de produção enfrenta alguns problemas, como manutenção do inventário e desafios na preparação de um plano de produção efetivo. O desenvolvimento de sistemas ERP se focou na resolução desses tipos de problema (Ou-Yang \& Chang, 2006).

Entre as aplicações os módulos de aplicações empresariais, o PDM e o ERP estão em posição de destaque nos departamentos de projeto e produção. O PDM auxilia os engenheiros a gerenciar os dados do processo de desenvolvimento do produto, e o ERP atua como a ferramenta principal para os processos relacionados a compras (order), produção e inventário (Ou-Yang \& Chang, 2006).

As informações geradas pelos sistemas PDM e ERP apoiam o processo de planejamento, que é chamado de CAPP (Computer-Aided Process Planning) e suportado por aplicações de software. Esse processo compreende o procedimento ou um conjunto de procedimentos que utiliza desenhos de engenharia, lista de materiais e outras especificações, como entrada para identificar e selecionar os processos, recursos, sequência de operações e parâmetros necessários 
para converter matéria-prima em produtos finalizados (Trotta, 2010; Siller et al., 2008).

Em relação à terceirização da manufatura, que demanda das modernas indústrias a utilização de sistemas via internet, os sistemas PDM legados (antigos) estão sendo migrados para sistemas baseados em tecnologia $W e b$, tornando-se uma ferramenta essencial para e-manufacturing. Os recentes avanços na tecnologia PDM tratam do uso da tecnologia $W e b$, porém, ainda existem limites no que se refere às disponibilidades, segurança, confiabilidade e escalabilidade para o mercado global (Sung \& Park, 2007).

\subsection{Projeto de produtos tendo em vista o descarte}

Segundo Yang et al. (2007), a maioria dos softwares tem como objetivo gerenciar as fases iniciais do ciclo de vida do produto, como a concepção, projeto do produto e preparação da manufatura, e muito poucos focam-se no ciclos finais de utilização do produto. Tendo em vista essa preocupação, foi desenvolvido um sistema PLM, para os consumidores da União Europeia, com o objetivo de gerenciar os dados oriundos da utilização doméstica dos produtos, podendo-se, dessa forma, obterem-se informações a respeito da distribuição, manutenção, utilização e fim de ciclo de vida do produto.

A preocupação em relação ao gerenciamento dessas informações se deve ao fato de que, após a Eco92, os consumidores se tornarem cada vez mais conscientes, o que demandou das organizações a utilização de práticas como o Eco-projeto, que é utilizado na Europa e está promovendo a difusão da reciclabilidade de veículos no mundo inteiro. A ação teve influência mesmo em países em desenvolvimento, como é o caso do Brasil, o que reflete nas políticas governamentais e também na legislação ambiental (Medina \& Naveiro, 2009).

Para atingir esses objetivos, Possamai \& Valentina (2007) propõem a criação de um indicador que leve em conta os níveis de montagem, a dificuldade de desmontagem e o uso de materiais fáceis de serem reutilizados.

Medina \& Naveiro (2009), por sua vez, sugerem a utilização de parcerias de longa duração, que são estabelecidas entre os fabricantes e seus fornecedores que trabalham em rede para compartilhar os riscos e os lucros, e também buscar soluções inovadoras para encorajar a reciclabilidade dos materiais.

Nesse cenário, a logística reversa ganha grande importância, apesar de, atualmente, a maior preocupação das empresas se relaciona ao trabalho logístico direto, entre suas plantas fabris e o consumidor final, envolvendo complexos sistemas de planejamento, de forma que todo o processo ocorra com precisão, objetivando a satisfação do cliente e a rentabilidade do negócio. Ao contrário do que muitos gestores pensam, o movimento inverso, ou seja, a logística reversa, não é apenas um processo de reciclagem de embalagens que, na maioria das vezes, devido à limitação de planejamento reverso, acaba sendo um grande gerador de custos (Gonçalves-Dias \& Teodósio, 2006).

\section{Materiais e métodos}

Para o desenvolvimento do estudo, foi efetuado um levantamento transversal (Babbie, 1999) com 397 profissionais do setor metal-mecânico, utilizando os grupos de discussão do Linkedin (rede social profissional). Assim, identificamos os seguintes perfis: profissionais que, preferencialmente, tenham participado em projetos de desenvolvimento de produtos, gestores e engenheiros de processos e produtos. Os membros da pesquisa foram entrevistados por meio de um questionário baseado na escala Likert (Hair et al., 2005) de 10 pontos. Os participantes atribuíam notas de discordância ou concordância em cada uma das 37 afirmativas que lhes foram apresentadas.

Os resultados foram tabulados, e para efetuar a análise foi utilizado o software SmartPls (Ringle et al., 2005), para a aplicação da análise PLS (Partial Least Squares), para a análise dos caminhos e para a composição do modelo final.

\section{Análise dos resultados}

A Figura 1 apresenta o modelo teórico que será avaliado utilizando-se a técnica de equações estruturais. As hipóteses são comentadas após a figura.

Devido ao fato de a gestão da colaboração precisar ocorrer durante todos os ciclos de vida do produto, do projeto inicial ao descarte, e também devido ao fato de o projeto verde, cuja grande preocupação é o desenvolvimento de produtos ambientalmente corretos e, por essa razão, precisar ser pensado de forma colaborativa, devendo fazer parte do planejamento estratégico da organização (Medina \& Naveiro, 2009), formula-se a Hipótese 1 desta pesquisa:

\section{H1: "O projeto verde" afeta a "Gestão da} Colaboração", favorecendo-a.

Devido aos aspectos apresentados por Possamai \& Valentina (2007), que discutem a importância da flexibilidade da manufatura, no que diz respeito ao desenvolvimento de produtos mais fáceis de desmontar e ao proposto por Scalice et al. (2009), no que diz respeito a estratégias que podem ser utilizadas pelos engenheiros de produto, a Hipótese 2 foi elaborada da seguinte forma:

H2: O "projeto verde" de produtos favorece a "Gestão da Manufatura", no que diz respeito a sua flexibilidade, criando produtos ambientalmente corretos.

Porque a gestão possibilita a troca de conhecimentos e a criação de redes de colaboração, por meio do uso de padrões que favorecem a utilização de ontologias (Nonaka et al., 2000), devendo ser utilizada em todas as fases do ciclo de vida de produtos, até mesmo no acompanhamento do uso destes pelos clientes 


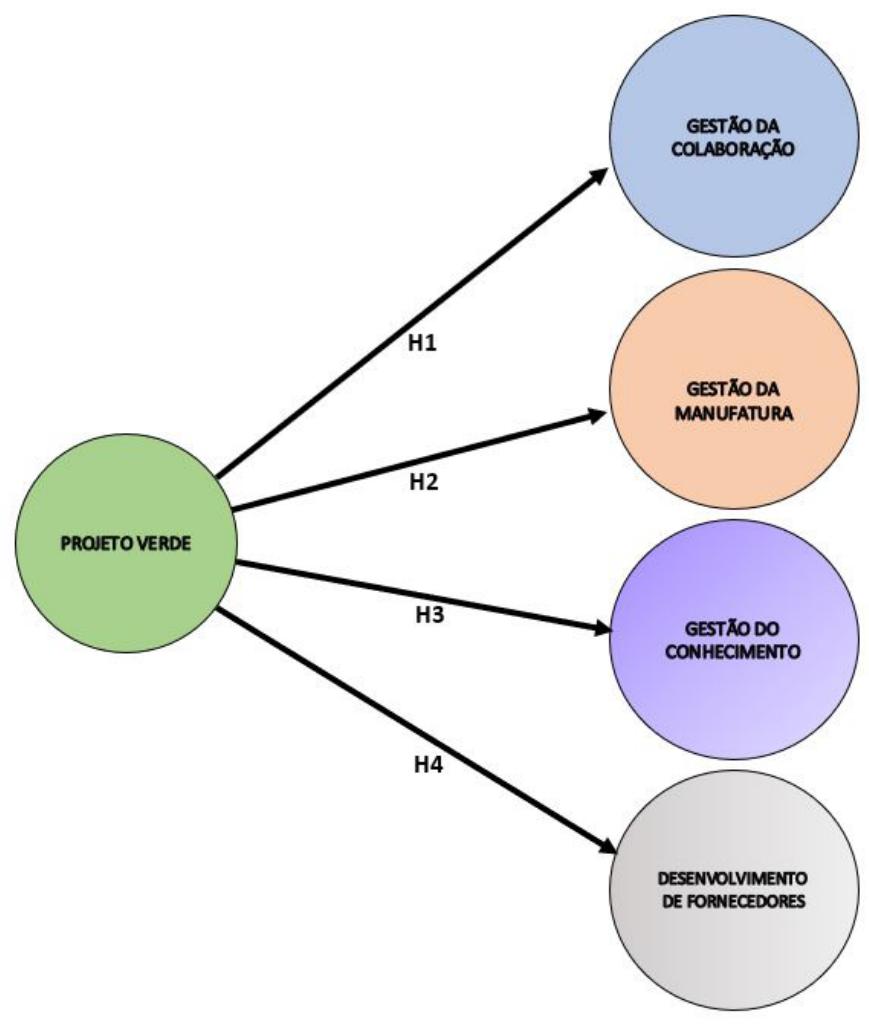

Figura 1. Modelo de Gestão do Ciclo de Vida de Produtos.

e utilização da rede de fornecedores (Kainuma \& Tawara, 2006), a Hipótese 3 foi formulada:

H3: O "projeto verde" de produtos favorece a "Gestão do Conhecimento", no que diz respeito ao compartilhamento de informações entre fornecedores e demais interessados no processo.

O projeto verde de produto é favorecido pelo uso de parcerias de longa data, conforme apontado por Kainuma \& Tawara (2006) e Leite (2005), no que diz respeito à logística reversa. Portanto, a Hipótese 4 foi a seguinte:

H4: O "projeto verde" de produtos é melhorado pelo desenvolvimento de fornecedores, favorecendo parcerias de longa data e facilitando a logística reversa.

Para a Análise PLS, foi utilizado o software "SmartPLS". Para isso, os dados em branco (não resposta) do questionário foram substituídos por -99. As variáveis também necessitaram ser reagrupadas para facilitar a análise.

As questões referentes aos fatores foram renomeadas para facilitar a análise dos dados.

Para a utilização da Análise PLS, foi utilizado o modelo proposto na Figura 1, o qual serviu de base para a geração do modelo de caminhos.

Primeiramente, foram analisadas as cargas fatoriais do chamado "outer model" (Ringle et al., 2005), por meio da análise das cargas das variáveis, desprezando-se valores abaixo de 0,5 . Por essa razão, as variáveis GECOL1, GECOL2, GECOL3, GECOL5, GECOL6 e GECOL7 foram eliminadas do fator "Gestão da Colaboração", a variável "DEFOR2", do fator "Desenvolvimento de Fornecedores", também foi eliminada, extinguindo o citado fator. Como a variável "DEFOR2" apresentou um valor muito alto e demonstrou possuir relação com o fator Gestão da Colaboração, foi transferida para esse fator.

Em seguida, o "algoritmo PLS" foi calculado novamente, gerando o modelo demonstrado na Figura 2:

O modelo apresentado na Figura 2 mostrou melhor adequação, pois as cargas das variáveis aumentaram, além de demonstrar maiores valores para o R-quadrado e para as cargas fatoriais (Hair et al., 2005) dos constructos.

Os coeficientes de caminho se mostraram muito adequados: "Projeto Verde" $\rightarrow$ "Gestão da Colaboração" = 0,655; "Projeto Verde" $\rightarrow$ Gestão da Manufatura $=0,722$ e "Projeto Verde" $\rightarrow$ Gestão do Conhecimento $=0,692$ (vide Figura 2). Esses valores mostram que há relações fortes entre os constructos (Hair et al., 2005), conforme pode ser verificado na Tabela 1.

Os $\mathrm{R}^{2}$ avaliam a porção das variáveis que explicam os constructos. Indica a qualidade do modelo ajustado. Valores de $0,75,0,50$ e 0,25 são considerados substanciais, moderados e fracos, respectivamente (Hair et al., 2014). 
A confiabilidade do constructo apresenta valores acima de 0,70 , indicando uma boa confiabilidade, enquanto o Alpha de Cronbach apresenta valores entre 0,6 e 0,8 (Hair et al., 2005), indicando que os constructos são válidos. Os valores de AVE se mostraram superiores ou muito próximos de 0,50 e, portanto, dentro do limite aceitável (Henseler et al., 2009). Ainda, as cargas fatoriais das relações entre os constructos e as variáveis se mostraram acima de 0,50 , indicando um bom ajuste do modelo geral (Henseler et al., 2009). Os resultados estão expostos na Tabela 2.

A Tabela 2 mostra as correlações existentes entre os fatores, indicando que o modelo escolhido apresenta bons índices de correlação, sendo o caminho escolhido o destacado (Chang, 2011). As raízes quadradas das AVEs se mostraram (destacadas em cinza na Tabela 2), com valores acima daqueles das correlações. Essa comparação mostra que o modelo geral tem uma boa validade discriminante (Fornell \& Larcker, 1981).

Para verificar a validade das variáveis latentes, ou seja, os constructos, utilizou-se o modo Bootstraping do SmartPLS 2.0 (Ringle et al., 2005), para que sejam significantes os valores dos testes t de Student dos coeficientes de regressão. O valor de 1,96 (nível de significância de 5\%) é muito adequado para se garantir que cada caminho (ou carga) foi considerado, tendo validade preditiva (Hayduk, 1987). Esses valores são demonstrados na Tabela 3.



Figura 2. Modelo Gestão do Ciclo de Vida de Produtos. Fonte: Dados da pesquisa (2012).

Tabela 1. Valores dos testes de ajuste do modelo.

\begin{tabular}{lcccc}
\hline & AVE & $\begin{array}{c}\text { Confiabilidade } \\
\text { Composta }\end{array}$ & R-Quadrado & $\begin{array}{c}\text { Alpha de } \\
\text { Cronbach }\end{array}$ \\
\hline Gestão da Colaboração & 0,532 & 0,773 & 0,475 & 0,560 \\
Gestão da Manufatura & 0,498 & 0,852 & 0,499 & 0,790 \\
Gestão do Conhecimento & 0,557 & 0,788 & 0,478 & 0,606 \\
Projeto Verde & 0,495 & 0,792 & --------- & 0,654 \\
\hline
\end{tabular}

Fonte: Dados da pesquisa (2012). 
A Tabela 4 mostra os valores de validade dos coeficientes de regressão do chamado "outer model", ou seja, as variáveis individuais que compõem a variável latente.

Pode-se constatar que todos os valores são significantes, pois os resultados obtidos nos testes $\mathrm{t}$ de Student são superiores a 1,92, indicando que, ao nível de significância de 0,05 , são significantes, o que confirma que as variáveis têm validade preditiva, segundo cada fator que elas compõem.

Por fim, foi calculado um índice geral de qualidade do modelo, denominado GoF (Goodness-of-fit), que é a média geométrica dos valores médios das AVEs e dos coeficientes de regressão do modelo SEM, resultando em 0,499 (Tenenhaus et al., 2005). O valor em questão é considerado muito apropriado, pois se preconiza o valor de 0,36 como adequado (Wetzels et al., 2009). Assim, o teste em questão mostra que o modelo geral está bem ajustado e atende o que se propôs a fazer na pesquisa em questão.

O Quadro 1 mostra as hipóteses que foram confirmadas e não confirmadas após a aplicação da análise PLS:

\section{Discussão dos resultados e comparação com modelos existentes}

O modelo proposto na Figura 2 nos remete à ideia de que, para que a organização consiga estabelecer a gestão do ciclo de vida de produtos, antes precisa adotar uma prática de melhor aproveitamento das

Tabela 2. Correlação entre os fatores e raízes quadradas das AVEs.

\begin{tabular}{lcccc}
\hline & $\begin{array}{c}\text { Gestão da } \\
\text { Colaboração }\end{array}$ & $\begin{array}{c}\text { Gestão da } \\
\text { Manufatura }\end{array}$ & $\begin{array}{c}\text { Gestão do } \\
\text { Conhecimento }\end{array}$ & Projeto Verde \\
\hline Gestão da Colaboração & 0,730 & & & \\
Gestão da Manufatura & 0,654 & 0,747 & & \\
Gestão do Conhecimento & 0,691 & 0,706 & 0,706 & \\
Projeto Verde & 0,689 & 0,720 & 0,691 & 0,701 \\
\hline
\end{tabular}

Fonte: Dados da pesquisa (2012).

Tabela 3. Validade do coeficiente de regressão das variáveis latentes.

\begin{tabular}{lc}
\hline & T Statistics (||O/STERR $)$ \\
\hline Projeto Verde -> Gestão da Colaboração & 6,097 \\
Projeto Verde -> Gestão da Manufatura & 3,894 \\
Projeto Verde -> Gestão do Conhecimento & 6,272 \\
\hline Fin
\end{tabular}

Fonte: Dados da pesquisa (2012).

Tabela 4. Validades dos coeficientes das relações entre variáveis e constructos.

\begin{tabular}{lc}
\hline \multicolumn{1}{c}{ Caminhos do modelo } & T Statistics (|O/STERR|) \\
\hline DEFOR1 <- Gestão da Colaboração & 4,073 \\
GECOL4 <- Gestão da Colaboração & 2,688 \\
GECOL8 <- Gestão da Colaboração & 4,168 \\
GECON1 <- Gestão do Conhecimento & 3,802 \\
GECON2 <- Gestão do Conhecimento & 3,794 \\
GECON3 <- Gestão do Conhecimento & 3,531 \\
GECON4 <- Gestão do Conhecimento & 2,244 \\
GEMAN1 <- Gestão da Manufatura & 4,168 \\
GEMAN2 <- Gestão da Manufatura & 2,534 \\
GEMAN3 <- Gestão da Manufatura & 3,781 \\
GEMAN4 <- Gestão da Manufatura & 2,060 \\
GEMAN5 <- Gestão da Manufatura & 2,455 \\
GEMAN6 <- Gestão da Manufatura & 3,634 \\
GEMAN7 <- Gestão da Manufatura & 3,335 \\
PROVER1 <- Projeto Verde & 3,811 \\
PROVER2 <- Projeto Verde & 3,261 \\
PROVER3 <- Projeto Verde & 3,742 \\
PROVER4 <- Projeto Verde & 4,320 \\
\hline
\end{tabular}

Fonte: Dados da pesquisa (2012). 
Quadro 1. Hipóteses Confirmadas/ Não Confirmadas.

\begin{tabular}{|c|c|c|}
\hline H1 & "O projeto verde" afeta a "Gestão da Colaboração", favorecendo-a. & Confirmada \\
\hline $\mathrm{H} 2$ & $\begin{array}{l}\text { O "projeto verde" de produtos favorece a "Gestão da Manufatura", no que diz } \\
\text { respeito a sua flexibilidade, criando produtos ambientalmente corretos. }\end{array}$ & Confirmada \\
\hline H3 & $\begin{array}{l}\text { O "projeto verde" de produtos favorece a "Gestão do Conhecimento", no que } \\
\text { diz respeito ao compartilhamento de informações entre fornecedores e demais } \\
\text { interessados no processo. }\end{array}$ & Confirmada \\
\hline H4 & $\begin{array}{l}\text { O "projeto verde" de produtos é melhorado pelo "desenvolvimento de } \\
\text { fornecedores", favorecendo parcerias de longa data, facilitando a logística } \\
\text { reversa. }\end{array}$ & Não Confirmada \\
\hline
\end{tabular}

Fonte: Elaborado pelo autor (2012).

matérias-primas e possuir consciência ambiental em suas ações. Porém, para que isso aconteça, o projeto verde precisa ser considerado nos estágios inicias do desenvolvimento do produto, na busca por materiais que possam ser reaproveitados e também na reciclagem de materiais existentes.

No entanto, Yang et al. (2007) já apontavam a necessidade de se desenvolver softwares PLM que se focam nos ciclos finas de vida do produto, o que levou a União Europeia a desenvolver um software para gerenciar os dados oriundos da utilização doméstica de produtos, porém, o modelo proposto demonstrou que essa preocupação já existe no Brasil.

A diferença do modelo anterior é que, além da preocupação em desenvolver produtos ambientalmente corretos, existe uma percepção de que essas práticas necessitam estar apoiadas por uma gestão da colaboração consistente, ou seja, sem a participação dos fornecedores, clientes, empresa, governo e demais stakeholders não é possível desenvolver um gestão do ciclo de vida consistente.

Para atingir o objetivo de desenvolver produtos ambientalmente corretos, apoiado por uma gestão do ciclo de vida de produtos, Possamai \& Valentina (2007) propõem um modelo que se foca na criação de um índice de dificuldade de desmontagem do produto. A grande diferença existente entre esse modelo e o proposto é que essa prática deve estar apoiada por uma gestão da manufatura consistente, pois deve aliar uma prática apresentada por Gerwin (2005), que sugere a melhoria da flexibilidade da manufatura, sendo que essa prática é importante para se apoiar projetos verdes, pois, na medida em que se propõe o uso de novos materiais, a área de manufatura precisa possuir capacidade de se adaptar às mudanças.

Para atingir esse objetivo, Qiu et al. (2007) propõem o uso de sistemas PLM, pois, segundo Trotta (2010), a importância desse tipo de sistema reside na capacidade de integrar a diversos outros tipos de sistemas, tais como CAx (CAD, CAM etc.), SCM, ERP's e produzir uma informação consistente e compartilhada por todos na organização, de modo que possa haver cooperação.
Para isso, uma gestão do conhecimento consistente é importante, pois permite às empresas integrarem todas as áreas de forma consistente, respeitando a ontologia de cada área, conforme sugerido por Nonaka et al. (2000), possibilitando a transferência do conhecimento. No entanto, para que a Gestão do Conhecimento seja efetiva, o modelo demonstrou que é importante estar apoiado por projeto verde, fazendo com que essa prática seja disseminada por toda a organização e esteja enraizada nas ações organizacionais e também nas de engenharia.

Para atingir esse objetivo, Fan \& Bermell-Garcia (2008) propõem o uso de KBE, fazendo com que os participantes do projeto possam avaliar a alteração de parâmetros e possam, dessa forma, colaborar e avaliar conjuntamente o uso e a aplicação de novos materiais, além de poderem desenvolver parcerias de longa duração com fornecedores, com o objetivo de se gerenciarem os ciclos finais de vida de produto.

O modelo proposto aponta que, para atingir a gestão do ciclo de vida de produto, é importante integrar projeto verde de produtos à gestão do produto em todos os ciclos, desde a concepção ao descarte. Para isso, é importante possuir práticas de gestão da colaboração, gestão da manufatura e gestão do conhecimento, distribuídos por toda organização e pela empresa estendida, de modo que haja cooperação e integração de todos os stakeholders.

A importância do modelo aqui proposto reside no fato de que já existe uma consciência ambiental, além de uma percepção geral de que os recursos do planeta precisam ser mais bem utilizados, para que as gerações futuras possam viver num modelo melhor do que o de hoje. A visão pessoal do pesquisador é a de que os empresários precisam desenvolver uma consciência de que produtos que se propõem a ser ambientalmente corretos. Além de agregarem valor e de preservarem o ambiente, esses produtos também melhoram a aceitabilidade do cliente, que já percebe a problemática gerada pela falta de consciência ambiental.

Medina \& Naveiro (2009) apontam que essa preocupação ficou ainda maior após a Eco92, ocorrida no Brasil, pois os clientes passaram a ter maior consciência, fato que demandou a busca da União 
Europeia por práticas como Eco-Projeto e aumento de reciclabilidade de veículos.

É obvio que essa consciência está apenas começando no Brasil, mas, com os resultados desta pesquisa, existem indicadores de que já está se disseminando entre os profissionais que trabalham na indústria mecânica e que, de alguma forma, possuem a percepção de que é necessário desenvolver produtos ambientalmente corretos e que possam ser reaproveitados.

A preocupação com o meio ambiente percebida nos clientes e em algumas empresas já está se refletindo em políticas governamentais e também na legislação ambiental, o que força as organizações a incluírem tais práticas já no planejamento estratégico (Medina \& Naveiro, 2009).

O aumento da conscientização ambiental dos clientes também pode trazer vantagens competitivas para as empresas que adotam esta política.

\section{Considerações finais}

O objetivo do artigo foi atingido ao propor um modelo de gestão do ciclo de vida de produto, o qual foi nomeado como PLM-PV3G, que é o acrônimo de Projeto Verde, que impulsiona três mecanismos de gestão, a saber: gestão da manufatura, gestão da colaboração e gestão do conhecimento. Nesse modelo, três elementos são importantes no modelo proposto: o primeiro é o uso de projeto verde desde o início do projeto de produtos até o descarte. O segundo elemento é a gestão da manufatura pelo uso de ferramentas como o CAD e o CAM, que permitem o compartilhamento das informações e decisões a respeito de mudanças e avaliação da manufaturabilidade de produtos. O terceiro elemento é a Gestão do Conhecimento, que deve permear todos os aspectos do desenvolvimento e melhora a criação de produtos, permitindo o uso das competências de todos os envolvidos no projeto. Porém, para que esse modelo funcione, deve ser apoiado por uma sólida gestão da colaboração, a construção de redes como parcerias entre os fornecedores, clientes e outros elementos da cadeia produtiva. Trata-se de um modelo que poderá ser adotado por empresas no mercado, pois apresenta, de forma consistente, os aspectos que envolvem a gestão do ciclo de vida de produtos.

As principais limitações do modelo são o fato de ter utilizado apenas profissionais da área de engenharia mecânica, o que não permitiu a validação do modelo em outros cenários; o fato de o questionário da pesquisa ter sido desenvolvido apenas em português também é um limitador da pesquisa, pois seria interessante o estudo em outras realidades, em outros países, aliás, essas lacunas podem ser utilizadas para o desenvolvimento de novas pesquisas.

As sugestões de novas pesquisas seriam a validação do modelo em outros cenários, como diferentes tipos de indústrias e com profissionais de outros níveis hierárquicos, por exemplo, gestores e diretores de empresas, pois já que a pesquisa foi totalmente desenvolvida com técnicos e engenheiros do setor metal-mecânico, também poderiam ser desenvolvidas pesquisas em outros países visando analisar a adequação da escala a novas realidades. Novos constructos e também novas variáveis poderiam ser adicionados ao questionário, a fim de adequá-lo às necessidades de pesquisadores, estudantes e empresários que estiverem interessados em desenvolver pesquisas nessa área.

\section{Referências}

Babbie, E. (1999). Métodos de pesquisas de survey. (Vol. 1). Belo Horizonte: UFMG.

Brigatini, J. A. D., \& Miguel, P. A. C. (2009). A preliminary proposal to improve product development process at an engine manufacturing company. Product: Management \& Development, 7(2), 161-169.

Brito, A. C., Ferreira, C. V., \& Sampaio, R. R. (2009). Communication management in virtual projects. Product: Management \& Development, 7(2), 171-176.

Chang, C. H. (2011). The Influence of user's trait on public e-service usage:a self-service technology perspective. Asian Social Science, 7(7), 3-11.

Cui, J., \& Qi, G. (2006). Research on integration technology for product lifecycle management system. In Proceedings of the VI International Conference on Intelligent Systems Design and Applications (pp. 1109-113). Jinan: IEEE.

Fan, I., \& Bermell-Garcia, P. (2008). International standard development for knowledge based engineering services for product lifecycle management. Concurrent Engineering, Research and Applications, 16(4), 271-277. http://dx.doi. org/10.1177/1063293X08100027.

Farouk, B., Troussier, N., Huet, F., Gidel, T., Bonjour, E., Eynard, B. (2008). PLM-based approach for collaborative design between OEM and suppliers: case study of aeronautic industry. Computer-Aided Innovation, 277(1), 157-168.

Fornell, C., \& Larcker, D. F. (1981). Evaluating structural equation models with unobservable variables and measurement error. JMR, Journal of Marketing Research, 18(1), 39-50. http://dx.doi.org/10.2307/3151312.

Gecevska, V., Chiabert, P., Anisic, Z., Lombardi, F., \& Cus, F. (2010). Product lifecycle management through innovative and competitive business environment. Journal of Industrial Engineering and Management, 3(2), 323-336. http://dx.doi.org/10.3926/jiem.2010. v3n2.p323-336.

Gerwin, D. (2005). An agenda for research on the flexibility of manufacturing process. International Journal of Operations \& Production Management, 25(12), 11711182. http://dx.doi.org/10.1108/01443570510633576.

Gonçalves-Dias, S. L. F., \& Teodósio, A. S. S. (2006). Estrutura da cadeia reversa:"caminhos" e "descaminhos" 
da embalagem PET. Produção, 16(3), 429-441. http:// dx.doi.org/10.1590/S0103-65132006000300006.

Hair, J. F., Anderson, R. E.; Tatham, R. L. \& Black, W. C. (2005). Análise Multivariada de dados. (5. ed. Vol. 1). Porto Alegre: Bookman.

Hair, J., Hult, G., Ringle, C., \& Sarstedt, M. (2014). A Primer on Partial Least Squares Structural Equation Modeling (PLM-SEM). Los Angeles: SAGE Publications.

Hayduk, L. A. (1987). Structural equation modeling with LISREL. Baltimore: The Johns Hopkins University Press.

Henseler, J., Ringle, C. M., \& Sinkovics, R. R. (2009). The use of partial least squares path modeling in international marketing. Advances in International Marketing, 20, 277-319.

Hou, J., Su, C., Zhu, L., \& Wang, W. (2008) Integration of $\mathrm{CAD} / \mathrm{PDM} / \mathrm{ERP}$ system based on collaborative design. In ISECS - International Colloquium on Computing Communication (pp. 561-566). Guagnzhow: ISECS.

Jinsong, Z., Qifu, W., Li, W., \& Yifang, Z. (2008). Configuration-oriented product modeling and knowledge management for made-to-order manufacturing enterprises. International Journal of Advanced Manufacturing Technology, 25(1-2), 41-52. http://dx.doi.org/10.1007/ s00170-003-1871-z.

Kainuma, Y., \& Tawara, N. (2006). A multiple attribute utility theory approach to lean and green supply chain management. International Journal of Production Economics, 101(1), 99-108. http://dx.doi.org/10.1016/j. ijpe.2005.05.010.

Kim, G. Y., Noh, S. D., Rim, Y. H., \& Mun, J. H. (2008). XML-based concurrent and integrated ergonomic analysis in PLM. International Journal of Advanced Manufacturing Technology, 39(9-10), 1045-1106. http:// dx.doi.org/10.1007/s00170-008-1400-1.

Lambert, S. C. (2010). E-infrastructure, science and Cris. Data Science Journal, 9, 1-6. http://dx.doi.org/10.2481/ dsj.CRIS7.

Lee, S. G., Ma, Y.-S., Thimm, G. L. \& Verstraeten, J. (2008). Product lifecycle in aviation repair and overhaul. Computers in Industry, 59(2-3), 296-303. http://dx.doi. org/10.1016/j.compind.2007.06.022.

Leite, P. R. Logistica Reversa: categorias e práticas empresariais em programas implementados no Brasil - um ensaio de categorização. In XXIX EnANPAD - Encontro da ANPAD. Brasília: ANPAD. 2005.

Livieiro, F., \& Kaminski, P. C. (2009). Managing transnational product development project teams. Product: Management \& Development, 7(1), 3-15.

Medina, H. V., \& Naveiro, R. M. (2009). Eco-design practices in Europe fostering automotive vehicles recyclability in Brasil. Product: Management \& Development, 7(1), 81-90.
Nantes, J. F. D., \& Lucent, A. R. (2009). Evaluation of product development process from technological innovation: a study in the segment of agricultural machinery and equipment. Product: Management \& Development, 7(2), 190-193.

Nisson, P., \& Fagerström, B. (2006). Managing stakeholder requirements in a product modeling system. Computers in Industry, 57(2), 167-177. http://dx.doi.org/10.1016/j. compind.2005.06.003.

Nonaka, I., Toyama, R., \& Konno, N. (2000). SECI, Ba and Leadership: a unified model of dynamic knowledge creation. Long Range Planning, 33(1), 5-34. http:// dx.doi.org/10.1016/S0024-6301(99)00115-6.

Ou-Yang, C., \& Chang, M. J. (2006). Developing an agentbased PDM/ERP collaboration system. International Journal of Manufacturing Technology, 30(3-4), 369-384. http://dx.doi.org/10.1007/s00170-005-0076-z.

Possamai, O., \& Valentina, L. V. O. D. (2007). A model of evaluation of design for disassembly. Product: Management \& Development, 5(2), 133-138.

Possamai, O., Valentina, L. V. O. D., \& Futami, A. H. (2007). Concurrent engineering: a good practice of product development in machine bureacracy organization. Product: Management \& Development, 5(1), 51-56.

Qiu, Z. M., Kok, K. F., Wong, Y. S., \& Fuh, J. Y. H. (2007). Role-based 3D visualization for assynchronous PLM collaboration. Computers in Industry, 58(8-9), 747755. http://dx.doi.org/10.1016/j.compind.2007.02.006.

Ringle, C., Wende, S., \& Will, A. (2005). SMARTPLS. Smarpls. Recuperado em 21 de janeiro de 2012, de www.smartpls.de

Rouibah, K., \& Caskey, K. R. (2003). Change Management in concurrent engineering from a parameter perspective. Computers in Industry, 50(1), 15-34. http://dx.doi. org/10.1016/S0166-3615(02)00138-0.

Rouibah, K., \& Oud-Ali, S. (2007). Dynamic data sharing and security in a collaborative product definition management system. Robotics and Computer-integrated Manufacturing, 23(2), 217-233. http://dx.doi.org/10.1016/j. rcim.2006.02.011.

Rozenfeld, H. \& Forcellini, F. A. (2006). Gestão do desenvolvimento de produtos: uma referência para a melhoria do processo. (1. ed.). São Paulo: Saraiva.

Santos, A. C. \& Forcellini, F. A. (2009). Effects of product development decision-making process on the supply chain. Product: Management \& Development

Scalice, R. K., Becker, D., \& Silveira, R. C. (2009). Developing a new compatibility table for design for recycling. Product: Management \& Development, 7(2), 141-148.

Sharma, A. (2005). Collaborative product innovation: integrating elements of CPI via PLM framework (Vol. 37, pp. 1425-1434). United States: Computer-Aided Design. 
Siller, H. R., Estruch, A., Vila, C., Abellan, J. V., \& Romero, F. (2008). Modeling workflow activities for collaborative process planning with product lifecycle management tools. Journal of Intelligent Manufacturing, 19(6), 689-700. http://dx.doi.org/10.1007/s10845-008-0120-6.

Su, S., \& Liu, Y. (2008). Research on Management of Ship Concurrent Collaborative Design based on PDM. In Proceding of the 2008 International Conference on Computer Science and Software Engineering (pp. 1922). Washington: [s.n.].

Sung, C. S., \& Park, S. J. (2007). A component-based product data management system. International Journal of Advanced Manufacturing Technology, 33(5-6), 614626. http://dx.doi.org/10.1007/s00170-006-0482-x.

Tang, D., \& Qian, X. (2008). Product lifecycle management for automotive development focusing on supplier integration. Computers in Industry, 59(2-3), 288-295. http://dx.doi.org/10.1016/j.compind.2007.07.002.

Tang, D., Eversheim, W., \& Shuh, G. (2004). A new generation of cooperative development paradigm in the toll and die making braching: strategy and technology. Robotics and Computer-integrated Manufacturing, 20(4), 301-311. http://dx.doi.org/10.1016/j.rcim.2003.11.001.

Tenenhaus, M., Vinzi, V. E., Chatelin, Y., \& Lauro, C. (2005). PLS Path Modeling. Computational Statistics \& Data Analysis, 48, 159-205. Recuperado em 15 de março de 2012, de https://studies2.hec.fr/jahia/webdav/ site/hec/shared/sites/tenenhaus/acces_anonyme/home/ art icles/PLS_PM_5.pdf

Thimm, G., Lee, S. G., \& Ma, Y. S. (2006). Towards unified modeling of product life-cycles. Computers in
Industry, 57(4), 331-341. http://dx.doi.org/10.1016/j. compind.2005.09.003.

Trotta, M. G. (2010). Product Lifecycle Management: Sustainability and Knowledge management as keys in a complex system of product development. Journal of Industrial Engineering and Management, 3(2), 309-322. http://dx.doi.org/10.3926/jiem.2010.v3n2.p309-322.

Venkatasubramanian, V. (2005). Prognostic and diagnostic monitoring of complex systems for product lifecycle management: Challenges and opportunities. Computers \& Chemical Engineering, 29(6), 1253-1263. http://dx.doi. org/10.1016/j.compchemeng.2005.02.026.

Wetzels, M., Odekerken-Schröder, G. \& van Oppen, C. (2009). Using PLS path modeling for assessing hierarchical construct models: guidelines and empirical illustration. Management Information Systems Quarterly, 33(1), 177-195. http://dx.doi.org/10.2307/20650284.

Yang, J., Goltz, M., \& Han, S. (2004). Parameter-based engineering: research and applications. Concurrent Engineering, Research and Applications, 12(4), 275286. http://dx.doi.org/10.1177/1063293X04041528.

Yang, X., Moore, P. R., Wong, C.-B., Pu, J.-S., \& Kwong Chong, S. (2007). Product lifecycle acquisition and management for consumer products. Industrial Management \& Data Systems, 107(7), 936-953. http:// dx.doi.org/10.1108/02635570710816685.

Zancul, E. S. (2009). Gestão do ciclo de vida de produtos: seleção de sistemas PLM com base em modelos de referência. (Tese de doutorado). Escola de Engenharia de São Carlos, Universidade de São Paulo, São Carlos. 